\title{
O dispositivo grupal como intervenção em reabilitação: reflexões a partir da prática em Terapia Ocupacional*
}

\section{The group device as intervention in rehabilitation: reflections from the practical one in Occupational Therapy}

\author{
Marisa Samea ${ }^{1}$
}

\begin{abstract}
SAMEA, M. O dispositivo grupal como intervenção em reabilitação: reflexões a partir da prática em Terapia Ocupacional. Rev. Ter. Ocup. Univ. São Paulo, v. 19, n. 2, p. 85-90, maio/ago. 2008.

RESUMO: As abordagens grupais têm sido, cada vez mais, fonte de investigação e intervenção na prática da terapia ocupacional. Este artigo busca discutir a utilização do dispositivo grupal como intervenção em reabilitação, levantando alguns elementos teóricos que fundamentam essa proposição. Um grupo terapêutico de atividades pode configurar-se como importante espaço para a explicitação e troca de diversos conteúdos relativos à reabilitação, como dificuldades, limites e potencialidades, vivenciados a partir de diferentes necessidades e demandas. Pode também, através dos processos vinculares, configurar-se em espaço para a construção de novos projetos de vida, singulares e coletivos.
\end{abstract}

DESCRITORES: Terapia ocupacional/tendências. Grupos de auto-ajuda/utilização. Reabilitação.

\footnotetext{
* Artigo extraído da Dissertação de Mestrado intitulada "Terapia Ocupacional e grupos: em busca de espaços de subjetivação" apresentada ao Programa de Pós-Graduação do Instituto de Psicologia da Universidade de São Paulo em 2002 para obtenção do título de Mestre em Psicologia. Área de concentração: Psicologia Social.

1. Terapeuta Ocupacional. Mestre em Psicologia Social pela USP. Especialista em coordenação de Grupos Operativos. Docente do Centro Universitário São Camilo e terapeuta ocupacional da Prefeitura do Município de São Paulo.

Endereço para correspondência: Marisa Samea. Centro Universitário São Camilo. Av. Nazaré, 1501. Ipiranga. São Paulo, SP. CEP: 04263-000. E-mail: marsamea@uol.com.br
} 
$\mathrm{A}$ produção teórica sobre as abordagens grupais em Terapia Ocupacional no Brasil é recente e ainda é restrita a poucos autores, havendo uma maior concentração dessas produções no campo da saúde mental. Entretanto, os grupos têm se revelado, cada vez mais, como importante dispositivo de investigação e intervenção na prática terapêutica. Este artigo pretende fundamentar e discutir algumas considerações sobre a possibilidade da utilização do grupo terapêutico como intervenção em reabilitação, a partir da prática em Terapia Ocupacional.

As observações de nossa prática grupal, o conhecimento de várias outras experiências onde o grupo tem sido utilizado com resultados bastante interessantes, os conhecimentos teóricos aliados às trocas entre os pares têm indicado a pertinência e relevância do estudo deste território como contribuição à prática clínica e social.

Para construir suas idéias e planificar ações sobre grupos, e desenvolver intervenções, a terapia ocupacional tem se reportado a conhecimentos que fazem fronteira com diversas áreas, entre elas a psicanálise, a psicologia social, a sociologia. Segundo Maximino (1997, p. 22), o uso de atividades feitas em ambiente grupal acompanha o desenvolvimento da própria história da terapia ocupacional, principalmente no seu aspecto de "transformadora" $e$ "reorganizadora" das instituições asilares.

Duncombe e Howe $(1985,1995)$ em duas investigações desenvolvidas com intervalo de 10 anos, demonstraram que $60 \%$ dos terapeutas ocupacionais utilizavam-se dos grupos em sua prática clínica.

No Brasil, destacamos os trabalhos das terapeutas ocupacionais Ballarin (1995), Benetton (1994), Constantinidis (2000), Ferrari (1990), Maximino (2001), entre outros, cuja produção teórica vêm trazendo importantes referenciais para o estudo de grupos. Em nossa pesquisa na Dissertação de Mestrado intitulada Terapia Ocupacional e grupos: em busca de espaços de subjetivação (2002), reportando-nos aos conhecimentos da área psicossocial, propusemos a utilização do grupo como dispositivo para a intervenção em reabilitação, fazendo a interlocução entre as produções do campo da saúde mental com aquelas da área das deficiências.

Na dissertação de mestrado fizemos o acompanhamento e estudo qualitativo de um processo grupal, com encontros duas vezes por semana ao longo de 7 meses, em instituição governamental de reabilitação. O grupo formado era heterogêneo, de adultos, alguns com deficiência outros com transtornos mentais, cuja constituição deu-se a partir do desejo de vivenciar a proposta, que era realizar atividades externas ao espaço institucional - como saídas à cidade - e também, de forma articulada, atividades plásticas - pintura, colagem, modelagem, entre outras - dentro da instituição.

\section{POR QUE GRUPOS?}

O grupo se constituiria como matéria-prima para a compreensão do sujeito, das articulações entre questões intrapsíquicas e as determinações concretas, situadas a partir do lugar deste sujeito na estrutura social (FERNANDES, 1994).

A concepção que nos norteia indica que os processos de constituição do sujeito acontecem através da produção intersubjetiva, onde estão implicados o mundo interno, as questões intra-psíquicas, portanto os processos inconscientes, e seu interjogo com a dinâmica vincular, ou seja,

a subjetividade é determinada histórica e socialmente enquanto o sujeito se constitui como tal no processo de interação entre os sujeitos, do qual o vínculo, como relação bicorporal, e o grupo, como rede vincular, constituem unidades de análise. (QUIROGA, 1984).

Pensamos no grupo como um veículo de produção de subjetividades, o que ele poderia captar e revelar em relação às marcas subjetivas de cada integrante e, em contraponto, o que ele ocultaria.

Segundo Barros, as oposições indivíduo-grupo, indivíduo-sociedade vêm no bojo da construção da categoria indivíduo presente desde o século XVI, ganhando destaque nos séculos XVIII e XIX, onde, no processo de "interiorização" o "individual" ganha um registro de sentidos que marca formas de estar, sentir, pensar e viver o mundo (BARROS, 1993, p. 147).

Este modo de funcionamento "individualizante" sobre os objetos e as práticas acabam por privatizar atos e afetos (p. 147). E a autora prossegue dizendo que:

o grupo, nesta perspectiva, vem a inserir-se como intermediário da relação indivíduo-sociedade. Duplica-se a dicotomia indivíduo-grupo e grupo-sociedade, na tentativa de se estabelecer uma passagem "mais suave" da compreensão dos fenômenos individuais aos sociais. O que se percebe é a insistência do "social" como algo "externo" ao individual mantendo-se a mesma lógica disjuntiva (interno-externo, indivíduo-grupo). (BARROS, 1993, p. 147).

Barros nos alerta que a noção de subjetividade não pode ser confundida com a de indivíduo, ressaltando que "ela é essencialmente fabricada e modelada no registro do social". (BARROS, 1993, p. 149).

O grupo pensado de forma individualizante fica colocado como intermediário entre o nível "individual" e o 
nível "social”. Mas, "se fizermos deslocar a noção de grupo para a noção de subjetividade, o que encontraremos será multiplicidade e provisoriedade" (BARROS, p. 151).

Neste sentido, os pequenos grupos nos parecem instrumentos privilegiados de investigação e intervenção no universo das populações marginalizadas e excluídas do tecido social, em um momento contemporâneo onde aspectos básicos como a cidadania, a ética e os direitos humanos se colocam em evidência e paradoxalmente apontam para os longos caminhos ainda a percorrer na direção de maior justiça social.

\section{O UNIVERSO DA REABILITAÇÃO}

Em nossa prática profissional, temos nos deparado com a imensa dificuldade de acesso e trânsito nos espaços sociais, públicos ou privados, das pessoas com deficiências e das pessoas com sofrimento psíquico, tanto por obstáculos concretos como barreiras arquitetônicas e dificuldades de acesso físico, como pelo lugar simbólico que tais populações ocupam nas redes de relações sociais. Seu lugar social aparece preponderantemente cristalizado, com poucas chances de mobilidade e se existe algum movimento este normalmente revela-se estereotipado, empobrecido. Suas histórias revelam imensas dificuldades e às vezes impossibilidade de acesso aos equipamentos sociais, quer sejam recursos de saúde, escolas, espaços culturais, locais de profissionalização, locais de trabalho, espaços de convivência e socialização. Esses sujeitos inserem-se na condição de desvantagem ou impedimento ${ }^{(1)}$, uma vez que vivenciam restrições das oportunidades de participação nas redes sociais. Assim, discutir, os comprometimentos mentais e as deficiências - e a multiplicidade de fatores a eles relacionados - nos conduz inevitavelmente ao universo da reabilitação.

No Brasil, as primeiras instituições especializadas no atendimento de pessoas com incapacidades físicas, sensoriais ou doenças mentais foram estruturadas na segunda metade do século XIX (SOARES, 1991). Uma longa e complexa trajetória ${ }^{(2)}$ marca os caminhos, avanços e dificuldades nas proposições do poder público e da esfera privada na atenção à reabilitação e é a partir dos anos 1990 que as produções teóricas acentuam a reflexão crítica e problematizam essa temática, repensando práticas e resultados. É nesse contexto que foi proposto o conceito de reabilitação psicossocial, que segundo a OMS, é um processo que facilita a oportunidade para indivíduos - que são deficientes, incapacitados ou limitados, por uma desordem mental - a encontrar seu nível ótimo de funcionamento independente na comunidade. Isso implica tanto aumentar competências individuais quanto introduzir mudanças ambientais de forma a criar uma vida da melhor qualidade possível para pessoas que tenham experenciado uma desordem mental que produz um certo nível de desvantagem. A Reabilitação Psicossocial objetiva estabelecer o nível ótimo de funcionamento de indivíduos e sociedades, e a minimização das desvantagens, incapacidades e deficiências, acentuando as escolhas individuais sobre como viver integrado dentro da comunidade. (OMS, 1996).

\section{GRUPOS EM REABILITAÇÃO: DISCUSSÃO}

No campo da reabilitação, as novas estratégias de atenção às populações com deficiência e com transtorno mental, buscam captar questões relativas à subjetividade, para além do olhar reducionista centrado na doença. Considerando o transtorno mental e a deficiência como fenômenos manifestos, com expressões no universo corporal e psíquico, que imprimem marcas na subjetividade, temos procurado ir ao encontro das representações daí advindas, e seus complexos desdobramentos, ao invés de buscar técnicas corretivas ou de normalização. É nesse contexto que a abordagem grupal configura-se, entre outras possibilidades, como dispositivo, espaço potencializador de encontros e contato com o outro, de questionamentos e indagações, de elaboração e trocas, de identificações, de confrontos.

Dentre as teorias sobre as abordagens grupais terapêuticas, tem norteado nossa prática e reflexão, principalmente, a teoria de Enrique Pichon-Rivière (1977, 1980, 1988, 1998). Este autor construiu sua trajetória da Psicanálise à Psicologia Social e elaborou um importante conjunto de conceitos sobre o universo do sujeito e dos campos inter-subjetivos e institucionais. Criador da técnica dos Grupos Operativos, Pichon-Rivière tem sido fonte de diversas produções teóricas e intervenções no campo da saúde, educação e práticas sociais e também no campo da Terapia Ocupacional.

Para Pichon-Rivière, o sujeito é o emergente de uma complexa trama de vínculos e relações sociais, é a síntese de uma história vincular e social. O sujeito é o agente, ator do processo interacional e ao mesmo tempo é determinado pelas relações que constituem suas condições concretas

\footnotetext{
(1) Segundo a OMS (1989), Impedimento ou desvantagem indicariam uma situação para um determinado indivíduo, em conseqüência de uma deficiência ou de uma incapacidade, que limita ou impede o desempenho de uma atividade que é normal em seu caso (em função de idade, sexo e fatores sociais e culturais).

(2) Para um estudo pormenorizado sobre a história da reabilitação no Brasil, recomendamos a leitura de Soares (1991) como referência.
} 
de existência. A partir da concepção de uma natureza humana social e historicamente determinada, o homem é compreendido como ponto de chegada de um processo histórico, como síntese de uma complexa rede de relações sociais.

Articular o conhecimento das abordagens grupais ao universo da Terapia Ocupacional tem-se constituído como desafio. O planejamento e a execução de atividades são instrumentos dos processos de intervenção em terapia ocupacional $e$ quando, no contexto do atendimento, vários sujeitos estão juntos compartilhando tempo, espaço e um fazer através das interações, múltiplos podem ser os conteúdos gerados pelos encontros, entrecruzamentos e conexões entre universos subjetivos diversos e singulares. $\mathrm{O}$ que é manifesto vem carregado de significações que, no contato com o outro, podem adquirir ainda novos significados, explicitando diferenças e semelhanças, traços culturais distintos, marcas das histórias e desejos.

A possibilidade da produção de uma "cadeia associativa via atividade" (MAXIMINO, 2001) pode contribuir para a compreensão dos modos de funcionamento relativos às histórias de cada um, aos vínculos, aos afetos, às formas de comunicação e interação, revelando os processos de subjetivação.

O espaço grupal possibilita o contato e o reconhecimento do próprio fazer, seus limites e facilidades; a observação do fazer do outro, a percepção de semelhanças e contrastes, e a potencialização do fazer junto. Nessa travessia, as múltiplas identificações e fantasias que entram em cena nos processos de interação conferem ao espaço grupal um contínuo movimento, um permanente interjogo, um efeito motor, no sentido de provocar e potencializar transformações. As produções podem favorecer o registro da memória dos processos, das vivências grupais e do que elas atualizam, oferecendo-lhes certa concretude.

Na bibliografia do campo da Terapia Ocupacional, há relatos sobre práticas grupais com pacientes com Parkinson (DAVIS, 1977, GAUTHIER et al., 1987), para deficientes físicos (VERSLUYS, 1980), para pacientes com traumatismo craniano (LUNDGREN; PERSECHINO, 1986), para pacientes com transtornos psicóticos (CONSTANTINIDIS, 2000; MAXIMINO, 2001). Neles a constituição grupal considera critérios nosológicos, com desenvolvimentos diversos e avaliações sobre os benefícios aos participantes. Em nossa prática com adultos, se trabalha com grupos heterogêneos ${ }^{(3)}$ não tomando o diagnóstico como critério central. Assim, a presença de alguma vulnerabilidade de saúde (como, por exemplo deficiência física, mental ou sensorial, sofrimento psíquico, HIV, entre outras) ou vulnerabilidade social (vítimas de violência, pessoas em situação de rua, entre outros) não são critérios de exclusão nem de configuração homogênea para a abordagem grupal. As proposições focam prioritariamente a atividade proposta, concepção essa pautada no conceito de tarefa de PichonRivière, tentando oferecer espaço de construção de sentidos para as múltiplas demandas surgidas a partir do trabalho com os sujeitos envolvidos.

Pichon-Rivière (1988) sustenta que não há vínculo, e conseqüentemente grupo, sem tarefa, já que em toda relação há um sentido de operatividade, alcançado ou não. $\mathrm{Na}$ técnica de Grupos Operativos, concebida por Pichon-Rivière os grupos definem-se como grupos centrados na tarefa. A tarefa poderia ser entendida como processo de elaboração, como conjunto de ações destinadas aos objetivos, a partir da necessidade e da transformação de uma ausência na direção daquilo que a satisfaz. A tarefa implica, então, em transformação da realidade externa e interna. O grupo, assim concebido, centra-se na relação que os integrantes mantêm com a tarefa.

O autor chama atenção para dois aspectos fundamentais de sua técnica: os aspectos manifestos ou explícitos e os aspectos implícitos ou latentes. Retomando a técnica analítica - fazer consciente o inconsciente - o autor propõe partir do explícito para descobrir o implícito, em um contínuo movimento em espiral dialética. A tarefa seria, então, a abordagem do objeto de conhecimento que apresenta um nível explícito ou manifesto, mas na qual também surgem algumas dificuldades, lacunas, cortes na rede de comunicação, que constituiriam os obstáculos para a sua realização - o nível implícito da tarefa. Os obstáculos ou dificuldades de abordagem, que supõem uma fantasia inconsciente (portanto implícita), denunciariam uma atitude de resistência à mudança. Para a teoria pichoniana, quanto maior a homogeneidade na tarefa e maior a heterogeneidade do grupo, maior a possibilidade de operatividade, ou seja de transformação da realidade e dos sujeitos envolvidos, em um movimento dialético.

Para Pichon-Rivière (1988), o conceito de grupo seria

conjunto restrito de pessoas, ligadas entre si por constantes de tempo e espaço e articuladas por sua mútua representação interna que se propõem, de forma explícita ou implícita a uma tarefa que constitui sua finalidade, interatuando através de complexos mecanismos de assunção e atribuição de papéis. (PICHON-RIVIÈRE, 1988, p.124)

(3) O trabalho com grupos heterogêneos tem sido uma prática norteadora nos Centros de Convivência e Cooperativas, unidades da Secretaria Municipal de Saúde de São Paulo. 


\section{CONSIDERAÇÕES FINAIS}

Com base nas concepções teóricas apresentadas, acreditamos que a abordagem grupal em terapia ocupacional teria a objetividade de uma tarefa, pautada no trabalho e elaboração dos desdobramentos advindos do encontro entre sujeitos que buscam reabilitação. Nela, os aspectos explícitos e implícitos podem ter lugar, em um processo de construção coletiva. A proposta de um grupo de terapia ocupacional deve considerar: as subjetividades envolvidas, em suas múltiplas e diversificadas cartografias - a história de cada sujeito, seus processos relacionais, seu cotidiano, seu contexto, possíveis rupturas sofridas ao longo da vida - sejam elas de ordem física, emocional ou social; as possibilidades de construção de perspectivas e novos projetos de vida; as questões institucionais e sociais que contextualizam os processos de reabilitação.

Há que se observar os papéis assumidos pelos sujeitos diante das vicissitudes de seu percurso, como conseguem inserir-se e transitar pela trama social, pesquisar e trabalhar com limites, dificuldades e cristalizações enfrentados. O grupo pode funcionar como disparador desses conteúdos, pois possibilita a explicitação de papéis assumidos e atribuídos e a vivência e exercício de novos papéis. À medida em que as interações acontecem, os vínculos se constroem e o grupo se constitui como espaço terapêutico, é possível perceber os movimentos de resistência, dificuldades de comunicação, conflitos entre os participantes, assim como os momentos de elaboração, de aprendizagem, de transformação dos sujeitos e de seus contextos - ou seja de operatividade e realização da tarefa.

No universo da reabilitação, o espaço grupal, pode gerar perspectivas inovadoras e a produção de novos sentidos e re-significações para os participantes. como pudemos verificar em nossa pesquisa.. Em nosso trabalho de mestrado, pudemos verificar o encontro com vários e importantes conteúdos - explícitos e implícitos - que possibilitaram o delineamento de várias leituras e instrumentalizaram o manejo grupal e as intervenções ao longo do processo. O espaço grupal promoveu o contato e trabalho com vários universos: cada sujeito envolvido (suas histórias e desejos); os vínculos construídos nessa trajetória (aproximações, identificações, conflitos); aspectos institucionais (atravessamentos, limites e possibilidades) e questões sociais (obstáculos visíveis, invisíveis e potencialidades). Percebemos também a gradativa construção de produções coletivas ancoradas nos processos de interação e vínculo, assim como o desdobramento de novos projetos de vida para cada um dos integrantes do grupo, ao final do processo.

A possibilidade da elaboração de alguns limites e obstáculos, de forma coletiva, e a vivência concreta de potencialidades oferecem aos sujeitos a oportunidade de maior conhecimento e apropriação de si mesmo, de sua condição e da realidade. Como lugar de diálogo, os grupos podem promover a construção de novas relações, na direção de maior autonomia e criação. Um processo grupal pode sensibilizar cada sujeito em sua singularidade, e provocar interessantes transformações em suas relações cotidianas - consigo mesmo, com os outros, com o mundo - conferindo-lhes movimento e aprofundamento, provocando um novo lugar - lugar de potência, de realização, de saber, de inclusão, para as populações geralmente marcadas pela falta, pelo não saber, pela exclusão.

Tal perspectiva pode contribuir na busca de novos horizontes e projetos de vida, no contexto do processo de reabilitação. Considerando a importância da utilização do dispositivo grupal em terapia ocupacional, acreditamos que essa proposição configura-se como mais uma das intervenções em reabilitação, somando-se a elas e possibilitando interlocuções com outros saberes e práticas na complexa rede de atenção e cuidados aos sujeitos. A prática e reflexão sobre os conjuntos inter-subjetivos podem oferecer a abertura, a ampliação do campo de ação e teorização nesse universo, estimulando novas pesquisas.

SAMEA, M. The group device as intervention in rehabilitation: reflections from the practical one in Occupational Therapy. Rev. Ter. Ocup. Univ. São Paulo, v.19, n. 2, p. 85-90, maio/ago. 2008.

\begin{abstract}
The group boardings have been, each time more, source of inquiry and intervention in the practical one of the occupational therapy. This article searchs to argue the use of the group device as intervention in rehabilitation, raising some theoretical elements that base this proposal. A therapeutical group of activities can be configured as important space for the revelation and changes of diverse relative contents to the rehabilitation, as difficulties, limits and potentialities, lived deeply from different necessities and demands. It also can, through the processes to tie, to configure themselves in space for the construction of new projects of life, singulars and collective.
\end{abstract}

KEY WORDS: Occupational therapy/trends. Self-help groups/utilization. Rehabilitation. 


\section{REFERÊNCIAS}

ABNT. NBR 9050, 1994. Acessibilidade de pessoas portadoras de deficiência a edificações, espaço, mobiliário e equipamento urbano. Rio de Janeiro: ABNT, 2001.

BALLARIN, M. L. G. S. Terapia ocupacional: uma perspectiva psicodinâmica (contexto e ilustração do atendimento de um grupo). Campinas, 1995. Dissertação (Mestrado), Universidade Estadual de Campinas.

BARROS, R. D. Benevides, Grupo e Produção. In: LANCETTI, A. (org.). SaúdeLoucura n.4 - Grupos e Coletivos, São Paulo: Hucitec, 1993.

BENETTON, M. J. A terapia ocupacional como instrumento nas ações de saúde mental. Campinas, 1994. 190p. Tese (Doutorado) - Programa de Pós-Graduação em Saúde Mental da Faculdade de Ciências Médicas da Universidade Estadual de Campinas.

BOUCHARD, V. C. Hemiplegic exercise and discussion group. Am. J. Occup. Ther., v. 26, n. 7, p. 330-331, 1972.

CONSTANTINIDIS, T. C. Possibilidades e limites na constituição de um grupo de terapia ocupacional com pacientes psicóticos: uma leitura a partir de Pichon-Rivière. São Paulo, 2000. Dissertação (Mestrado) - Instituto de Psicologia, Universidade de São Paulo.

DAVIS, J. C. Team management os Parkinson`s disease. Am. J. Occup. Ther., v. 31, n. 5, p. 300-308, 1977.

DUCOMBE, L. W.; HOWE, M. C. Group work in occupational therapy: a survey of practice. Am. J. Occup. Ther., v. 39, n. 3, p. $163-170,1985$.

DAVIS, J. C. Group treatment: goals, tasks, and economic implications. Am. J. Occup. Ther., v. 49, n. 3, p. 199-205, 1995.

FERRARI, S. M. L.; AGUIRRE, B. Aspectos do funcionamento da clínica de grupos e sua especificidade na terapia ocupacional. Bol. Psiquiatria, São Paulo, p. 22-23, jan 89/1990.

FERNANDES, M. I. A. A subjetividade à luz de uma teoria de grupos. Psicologia USP, v.5, n. 1/2, 1994.

GAUTHIER, L.; DALZIEL, S.; GAUTHIER, S. The benefits of group occupational therapy for patients with Parkinson's disease. Am. J. Occup. Ther., v. 41, n. 6, p. 360-365, 1987.

LUNDGREN, C.; PERSECHINO, E. Cognitive group: a treatment program for head-injured adults. Am. J. Occup. Ther., v. 40, n.
6, p. $397-401,1986$

MAXIMINO, V. S. Grupos de atividade com pacientes psicóticos. São José dos Campos: UniVap - Universidade do Vale do Paraíba, 2001.

NEISTADT, M. E. An occupational therapy program for adults with developmental disabilities. Am. J. Occup. Ther., v. 41, n. 7, p. 433-438, 1987.

PICHON-RIVIÈRE, E. O conceito de ECRO. In: Temas de psicologia social, n. 1, Buenos Aires, 1977.

PICHON-RIVIÈRE, E. Historia de la tecnica de los grupos operativos. In: Temas de psicologia social, n. 3. Buenos Aires, 1980.

PICHON-RIVIÈRE, E. O processo grupal. $3^{\text {a }}$ ed. São Paulo: Martins Fontes, 1988.

PICHON-RIVIÈRE, E. Teoria do vínculo. $6^{\mathrm{a}}$ ed. São Paulo: Martins Fontes, 1998.

PICHON-RIVIÈRE, E.; QUIROGA, A. P. Psicologia de la vida cotidiana. Buenos Aires: Nueva Visión, 1985.

PROGRAMA DE AÇÃO MUNDIAL PARAAS PESSOAS COM DEFICIÊNCIA. Brasília: Ministério da Justiça, 1997.

PSYCHOSOCIAL REHABILITATION: A CONSENSUS STATEMENT. Division of Mental Health and Prevention of Substance Abuse, Genebra, 1996.

QUIROGA, A. P. La concepcion del sujeto en el pensamiento de Enrique Pichon-Rivière. Rev. Asoc. Psicoanal. Argentina, v. 3, 1984.

SAMEA, M. Terapia ocupacional e grupos: em busca de espaços de subjetivação. São Paulo, 2002. 184 p. Dissertação (Mestrado) - Instituto de Psicologia da Universidade de São Paulo.

SECRETARIADO NACIONAL DE REABILITAÇÃO/OMS. Classificação internacional das deficiências, incapacidades e desvantagens (handicaps): uma manual de classificação das conseqüências das doenças. Lisboa: Secretariado Nacional de Reabilitação, 1989.

SOARES, L. B. T. Terapia ocupacional: lógica do capital ou do trabalho? São Paulo: Hucitec, 1991.

VERSLUYS, H. P. The remediation of role disorders through focused group work. Am. J. Occup. Ther., v.46, n. 6, p. 541$545,1980$. 